\title{
Energías de Interacción y Geometrías de Equilibrio para Aglomeraciones de 1,2-propanodiol con 3,4, 5 y 6 Moléculas de Agua
}

\author{
Adolfo E. Ensuncho ${ }^{(1)}$, Jesús M. López ${ }^{(1)}$ y José G. Carriazo ${ }^{(2)}$
}

(1) Universidad de Córdoba, Departamento de Química, Cra 6 № 76-103, Montería,

Córdoba-Colombia (e-mail: aensuncho@sinu.unicordoba.edu.co; jmanuellopez.gqc@gmail.com),

(2) Universidad Nacional de Colombia, Departamento de Química, Cra 30 N 45-03,

Bogotá, D.C., Colombia (e-mail: jcarriazog@unal.edu.co)

Recibido Feb. 15, 2011; Aceptado Abr. 19, 2011; Versión Final recibida May. 03, 2011

\section{Resumen}

Se reportan las geometrías de equilibrio y energías de interacción de aglomeraciones (clusters) formadas por los sistemas 1,2-propanodiol con 3, 4, 5 y 6 moléculas de agua. Esto con el fin de avanzar en la comprensión de las interacciones que predominan a nivel molecular en compuestos tipo dioles alifáticos con agua. Las aglomeraciones fueron generadas estocásticamente, seleccionadas mediante una prueba de aceptación metrópolis modificado implementado en el programa Recocido Simulado (Simulated Annealing) con Energía Cuántica y optimizados con el nivel de teoría B3LYP/6-31G(d). Las diferentes configuraciones generadas se caracterizaron por la formación preferencial de geometrías cíclicas. En estas geometrías, los enlaces de hidrógeno primario jugaron un rol fundamental en la estabilidad y probabilidad de existencia de estos agregados moleculares.

\section{Interaction Energies and Equilibrium Geometries for Clusters 1,2-propanediol with 3, 4, 5 and 6 Water Molecules}

\begin{abstract}
The equilibrium geometries and interaction energies in clusters of 1,2-propanediol with 3,4,5 and 6 water molecules were evaluated. This was done to better comprehend the predominant interactions existing at molecular level in aliphatic diols with water. The clusters were stochastically generated and selected by using a modified metropolis acceptance test implemented in the Simulated Annealing program with Quantum Energy software. The geometry of these systems was optimized by B3LYP/6-31G(d) calculations. The different generated configurations were characterized by the preferential formation of cyclic geometries. In these geometries, the primary hydrogen bonds have a fundamental role on the stability and probability of existence of these molecular aggregates.
\end{abstract}




\section{INTRODUCCIÓN}

Las mezclas agua/alcoholes alifáticos del tipo diol son usados como cosolventes en la industria farmacéutica para incrementar la solubilidad en solución acuosa de algunos formas farmacéuticas homogéneas tales como jarabes, emulsiones, elíxires entre otros; el 1,2-propanodiol se usa hoy día en la preparación de estas formulaciones (Swarbrick, 2002; Buggins et al., 2007). Debido al interés creciente en la investigación de estos sistemas, se han venido desarrollando estudios fisicoquímicos de las mezclas diol/agua, especialmente, a través de la descripción de sus propiedades termodinámicas, obteniéndose resultados que han constituido un criterio clave en el diseño de dosis farmacéuticas adecuadas (Pérez et al., 2003; Jiménez y Martínez, 2005). Sin embargo aunque las propiedades macroscópicas de estas mezclas han sido cuidadosamente estudiadas (Romero et al., 2007), no hay claridad suficiente sobre las características estructurales y energéticas de las posibles interacciones que a nivel nanométrico se originan. Consecuentemente, durante las últimas dos décadas el amplio uso de herramientas experimentales modernas (Dixit et al., 2002) ha permitido obtener valiosa información sobre la nano-estructura de soluciones acuosas que contienen alcoholes.

Asimismo, la disponibilidad de ordenadores con alta capacidad de computo y sofisticados métodos de simulación molecular, han brindado un gran aporte en el entendimiento de tales interacciones (Ruckenstein et al., 2005). Así, se reportan estudios computacionales, donde se describe que las interacciones que típicamente ocurren en estos sistemas son debidas a enlaces de hidrógeno (Mejía et al., 2007, 2010). Estas interacciones intermoleculares son la base para explicar la formación de clústeres (David et al., 2009) y su influencia sobre las propiedades macroscópicas mensurables para estas mezclas; razón por la cual las mezclas alcohol/agua interactúan simultáneamente formando enlaces de hidrógeno primarios y secundarios (Oliveira y Vasconcellos, 2006). En este contexto, el estudio a nivel molecular de la formación de clústeres del compuesto 1,2-propanodiol con 3, 4, 5 y 6 moléculas de agua, se realizó con el propósito de contribuir al entendimiento limitado de las interacciones moleculares originadas para este tipo de mezclas diol/agua.

\section{METODOLOGÍA}

Para la generación de los clústeres moleculares se utilizó programa ASCEC (Annealing simulado con energía cuántica) (Pérez et al., 2008), el cual implementa una versión adaptada del algoritmo de optimización Annealing Simulado (AS). El programa ASCEC realiza una búsqueda estocástica en el espacio conformacional del clúster molecular, evaluando la energía cuántica de cada conformación mediante un programa externo, en este caso particular se usó el paquete computacional Gaussian03 (Frisch, 2004). Una ejecución típica del programa ASCEC, requiere los siguientes parámetros: Una ruta de enfriamiento, geometría inicial en coordenadas cartesianas, longitud del cubo en el que el sistema estará envuelto, número máximo de estructuras a evaluar a cada temperatura (MaxCyc), máximo desplazamiento permitido y cambios de orientación para las moléculas individuales dentro de la estructura del clúster, y finalmente combinación Hamiltoniano/base para calcular la energía. Se utilizó el hamiltoniano semi-empírico PM3 (Kong et al., 2000), para calcular las energías durante la ejecución del ASCEC.

Las geometrías generadas se analizaron con base al criterio establecido por un test de aceptancia, para el cual si un cambio estructural disminuye la energía $\Delta E<0$, entonces la estructura es aceptada, y en el caso que $\Delta \mathrm{E}>0$, la nueva estructura solo se acepta sí pasa el test modificado de Metropolis (Pérez et al., 2008), $\Delta E / E_{j}<\exp \left(-\Delta E / k_{B} T\right)$, donde j es la estructura bajo evaluación. En el caso que la estructura no satisfaga esta condición, nuevamente se modifica aleatoriamente. Para evitar un gran número de evaluaciones de la energía no significativas, se genera solamente un número máximo de estructuras a cada temperatura mediante la reducción del parámetro de máximo desplazamiento permitido (MaxCyc) cada vez que es alcanzado.

La ejecución del programa ASCEC genera un grupo de estructuras candidatas a las cuales se les optimiza posteriormente por métodos analíticos. Para la optimización y caracterización de las estructuras generadas por el programa ASCEC, se utilizó el funcional de la densidad híbrido 
B3LYP (Koch, 2001), en combinación con un conjunto base 6-31G(d). Se caracterizaron los puntos estacionarios sobre la superficie de energía potencial por el método B3LYP (no eingevalores negativos de la matrix del Hessiano) mediante cálculos de frecuencia (Cuevas, 2003). La población de isómeros, $x_{i}$, sobre una sección determinada dentro de la superficie de energía potencial (Restrepo, 2005) se estimó a través de la ecuación (1).

$\% x_{i}=g_{i} e^{-E i / k B T} / \Sigma_{i} g_{i} e^{-E i / k B T}$

Donde $\mathrm{g}_{\mathrm{i}}$, es la degeneración del isómero $\mathrm{i}, \mathrm{k}_{\mathrm{B}}$ es la constante de Bolztmann, $\mathrm{T}$, la temperatura absoluta y $E_{i}$ la energía calculada por el funcional B3LYP. Todas las optimizaciones, frecuencias, así como los cálculos de energía se llevaron a cabo con el paquete computacional Gaussian03. Las energías de enlace por molécula (BE/molécula) se calcularon mediante la ecuación (2):

$\mathrm{BE} / \mathrm{Molec}=\left(n E_{i}-E_{n}\right) / n$

Donde $\mathrm{nE}_{\mathrm{i}}$ corresponde a la suma de la energías individuales de cada una de las moléculas que forman el clúster, $E_{n}$ es la energía total del clúster y $n$ es el número total de moléculas (Pérez et al., 2008). Las energías relativas $(\Delta \mathrm{E})$ se calcularon como la diferencia de energía de cada estructura con la de mínima energía (Grabowski et al., 2005). Las estructuras de los clústeres fueron nombradas como $\mathrm{ns}_{\mathrm{i}}$; donde $n$ indica el número de moléculas de agua y el subíndice $\mathrm{i}$ indica un arreglo geométrico en particular.

\section{RESULTADOS Y DISCUSIÓN}

Se construyeron las geometrías iniciales de los respectivos clústeres a través del enfoque descrito anteriormente, situando la molécula de 1,2-propanodiol con 3, 4, 5 y 6 moléculas de agua respectivamente; en un mismo punto, posición y orientación permitiendo que se desarrollaran bajo las condiciones del Annealing dentro de un cubo de $8 \AA$ de longitud. Para calcular las energías de las configuraciones de los clústeres generados aleatoriamente, se usó el hamiltoniano semiempírico PM3. La ruta de enfriamiento seguida fue con una temperatura inicial de $500 \mathrm{~K}$, con un decrecimiento del $10 \%$ y un total de 300 temperaturas.

El sistema de mayor número de clústeres generados en el proceso de búsqueda estocástica usando ASCEC, fue el 1,2-propanodiol con 3 moléculas de agua, con un total de 124 clústeres, de los cuales 7 convergieron a la misma estructura después de optimizar con B3LYP/6-31G(d). La tabla 1, presenta las configuraciones con una concentración de población de isómeros significativa y orden decreciente de estabilidad, correspondieron a los clústeres, 3s97, 3s109, 3s75, 3s102, 3s48, 3s87 y 3s101, respectivamente. Teniendo en cuenta las energías de enlace por molécula, energías relativas y concentración de isómeros $\left(\% \mathrm{x}_{\mathrm{i}}\right)$ sobre la superficie de energía potencial evaluada con el método B3LYP.

La estructura 3s97 presentó la mayor población isómeros con un 27.97\%. La figura 1, presenta el clúster 3s97 que corresponde al mínimo global. Algunas características notables de esta configuración es que todos los enlaces de hidrógeno son primarios (del tipo $\mathrm{HO} \cdots \mathrm{H}$ ), formando estructuras cíclicas, con 3 enlaces $\mathrm{O}-\mathrm{H}$ exocíclicos, y sus ángulos de enlace tienden hacia la colinealidad (ángulos cercanos a $180^{\circ}$ ), lo cual, maximiza las fuerzas atractivas y minimiza las repulsiones (Reichardt, 2003), permitiendo que la estructura sea más estable.

La figura 2, presenta las geometrías optimizadas para las configuraciones 3s109, 3s75 y 3s102 respectivamente. Aunque estas configuraciones presentan probabilidades de formarse levemente inferiores a la configuración $3 \mathrm{~s} 97$, los valores de energía de enlace por molécula son menores (tabla 1) y por tanto la fuerza de los enlaces de hidrógeno también disminuye. 

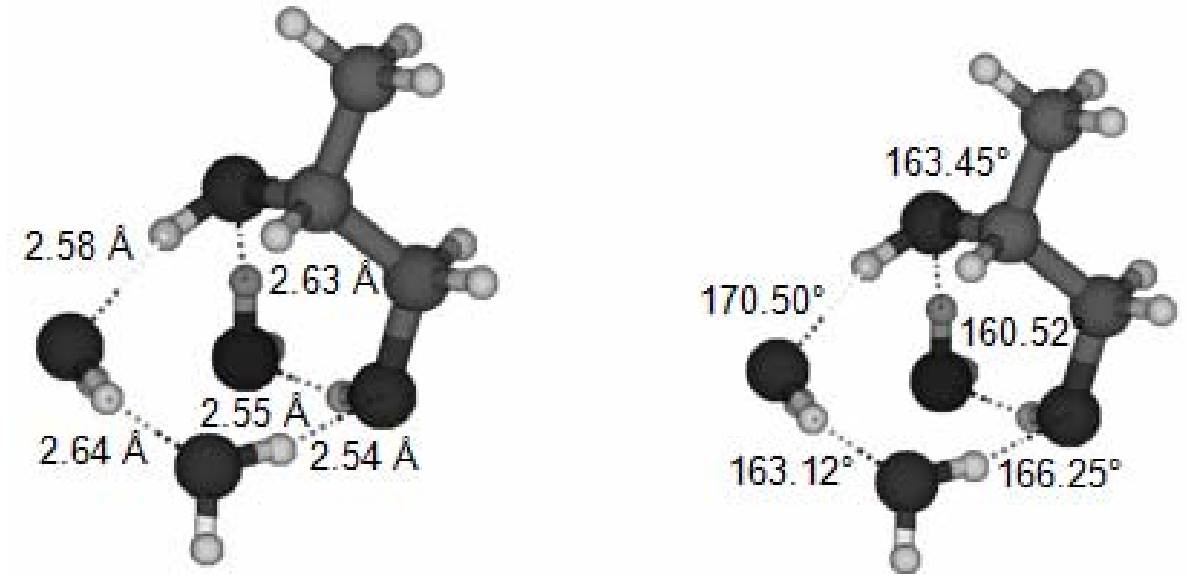

Fig. 1: Geometría del mínimo global, clúster 3s97: 1,2-propanodiol con 3 moléculas de agua. Código de colores/átomos: Negro: O; Gris: C; Blanco: H

Tabla 1: Clústeres de 1,2-propanodiol con 3 moléculas de agua y $\% x_{i}$

\begin{tabular}{cccc}
\hline Clúster & $\mathrm{BE} / \mathrm{Molec}\left(\mathrm{kcal} \mathrm{mol}^{-1}\right)$ & $\Delta \mathrm{E}\left(\mathrm{kcal} \mathrm{mol}^{-1}\right)$ & $\% \mathrm{x}_{\mathrm{i}}$ \\
\hline 3s97 & 12.35 & 0.00 & 27.97 \\
3s109 & 12.28 & 0.28 & 17.78 \\
3s75 & 12.24 & 0.43 & 13.37 \\
3s102 & 12.20 & 0.58 & 10.68 \\
3s48 & 12.16 & 0.76 & 8.00 \\
3s87 & 12.14 & 0.82 & 7.03 \\
3s101 & 12.13 & 0.89 & 6.19 \\
\hline
\end{tabular}

$3 s 109$
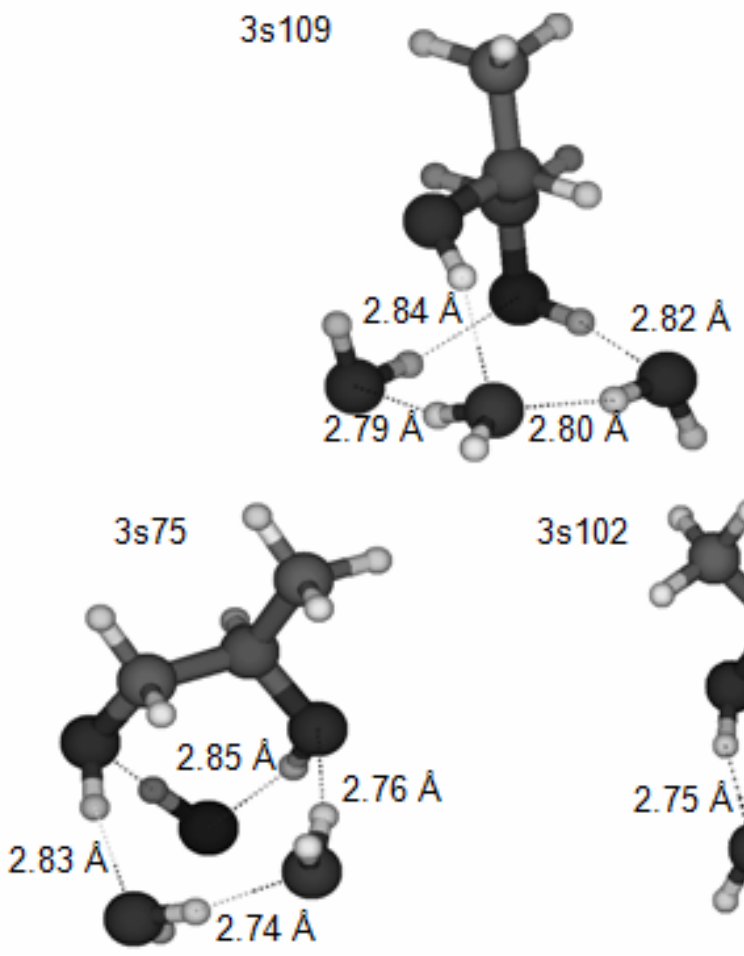

$3 s 102$

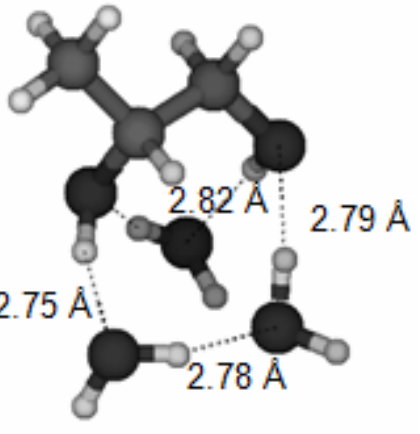

Fig. 2: Configuraciones 3s109, 3s75 y 3s102 
Para el sistema 1,2-propanodiol con 4 moléculas de agua, sólo seis clústeres presentaron poblaciones \%xi diferentes de cero. La tabla 2, resume los valores de energía de enlace por molécula, energías relativas y concentración de isómeros (\%xi) para cada clúster formado.

Tabla 2: Clústeres para 1,2-propanodiol con 4 moléculas de agua

\begin{tabular}{cccc}
\hline Clúster & $\begin{array}{c}\mathrm{BE} / \mathrm{Molec} \mathrm{kcal} \\
\mathrm{mol}^{-1}\end{array}$ & $\Delta \mathrm{E} \mathrm{kcal} \mathrm{mol}$ & $\% \mathrm{Xi}$ \\
\hline $4 \mathrm{~s} 48$ & 13.21 & 0.00 & 45.49 \\
$4 \mathrm{~s} 10$ & 13.01 & 0.66 & 15.27 \\
$4 \mathrm{~s} 16$ & 13.08 & 0.66 & 15.27 \\
$4 \mathrm{~s} 17$ & 13.07 & 0.68 & 15.27 \\
$4 \mathrm{~s} 5$ & 12.94 & 1.34 & 5.13 \\
$4 \mathrm{~s} 26$ & 12.94 & 1.58 & 3.27 \\
$4 \mathrm{~s} 15$ & 7.34 & 29.34 & 0.0 \\
\hline
\end{tabular}

En la figura 3, se presentan las geometrías de los clústeres de mínima y máxima energía de 1,2propanodiol con 4 moléculas de agua que corresponden a las configuraciones $4 \mathrm{~s} 48$ (3A) y $4 \mathrm{~s} 15$ (3B) respectivamente. Al comparar las configuraciones $4 \mathrm{~s} 48$ (mínimo global) y 4s15, se observa que las preferencias geométricas así como la alta estabilidad de la configuración $4 \mathrm{~s} 48$ es una consecuencia directa de la mayor interacción (afinidad) entre las moléculas de agua y el diol, que en la configuración 4s15 no se observa (figura 3B). La geometría del arreglo 4s48 evidencia que los enlaces de hidrógeno primario del tipo $\mathrm{HO} \cdots \mathrm{H}$ tienen menor distancia que los que se forman en la estructura 4s15 esto podría explicarse por la pobre interacción entre las moléculas de agua y el diol, que se refleja en la nula concentración de isómeros $\left(\% \mathrm{x}_{\mathrm{i}}=0\right)$ para esta estructura.

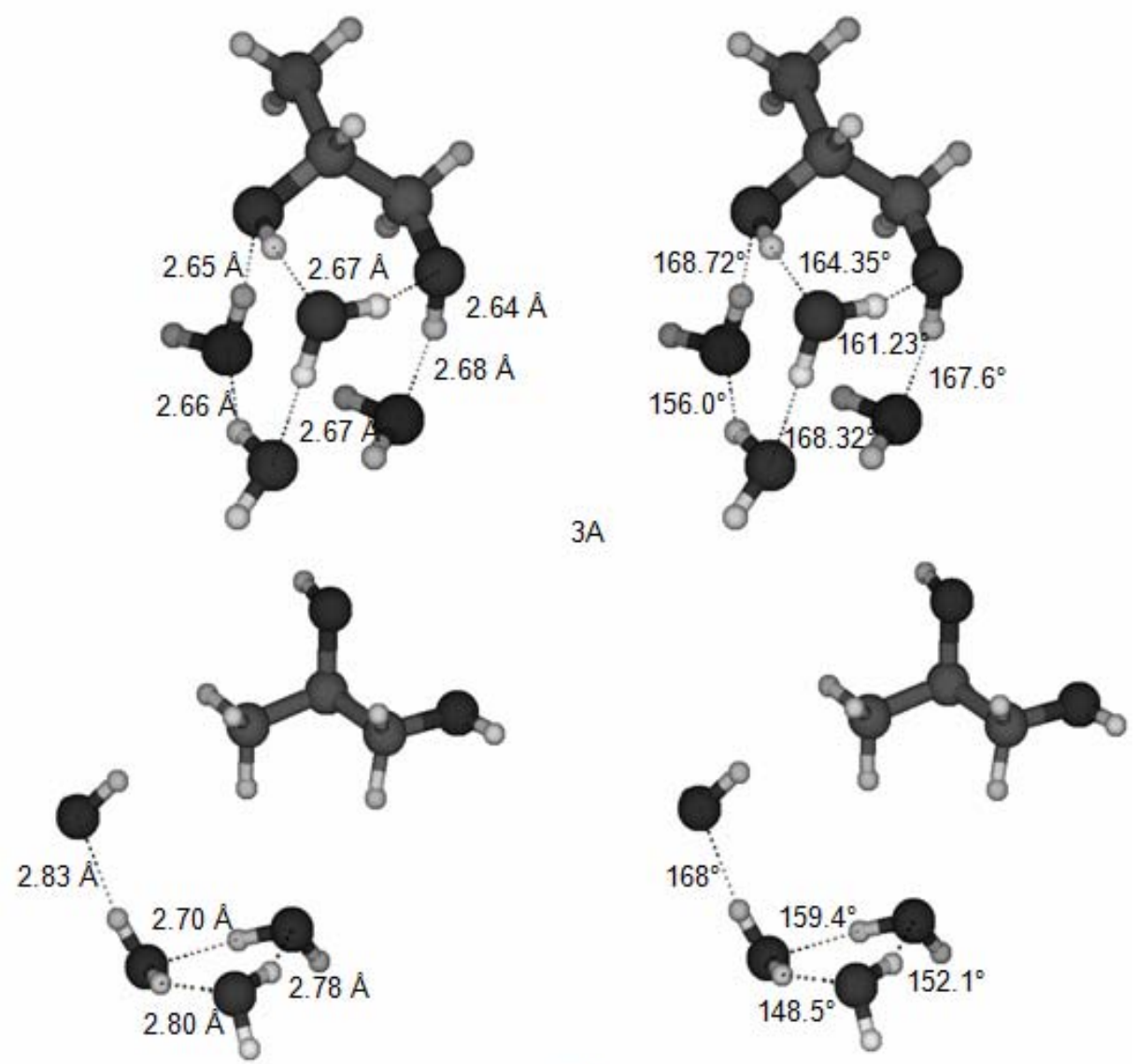

$3 \mathrm{~B}$

Fig. 3: Clústeres de mínima y máxima energía para el sistema 1,2-propanodiol con 4 moléculas de agua. Distancias $\mathrm{HO} \cdots \cdot \mathrm{H}$ en angstrom $(\AA)$ y ángulos de enlace $\mathrm{O}-\mathrm{H}-\mathrm{O}$ en grados $\left({ }^{\circ}\right)$ 
En la medida que aumenta el número de moléculas de agua prevalece la formación de enlaces de hidrógeno primarios. La figura 4, presenta los clústeres de mínima y máxima energía para el sistema de 1,2-propanodiol con 5 moléculas de agua designados como 5s22 (4A) y 5s9 (4B) respectivamente.

En la geometría de $5 \mathrm{~s} 22$ (mínimo global), las distancias de los enlaces de hidrógeno $\mathrm{HO} \cdots \mathrm{H}$ son menores que en $5 \mathrm{~s} 9$, implicando con esto la interacción preferencial de las moléculas de agua con los grupos $\mathrm{O}-\mathrm{H}$ del 1,2-propanodiol sobre las interacciones entre las moléculas de agua, en la formación de trímeros de moléculas de agua (figura 4B). También se observa en la configuración 5 s9 que hay pocas interacciones con 1,2-propanodiol, explicando esto la probabilidad nula de existencia para este clúster y una energía relativa de $16.97 \mathrm{kcal} \mathrm{mol}^{-1}$ veces mayor que $5 \mathrm{~s} 22$. Igualmente, para los clústeres con 6 moléculas de agua (figuras $5 \mathrm{~A}$ y $5 \mathrm{~B}$ ), sigue una tendencia muy similar a lo mostrada con 5 moléculas de agua, se resalta que para el clúster de máxima energía designado como $6 \mathrm{~s} 13(5 \mathrm{~B})$ hay un patrón geométrico similar a $5 \mathrm{~s} 9$, solo que en lugar de formar trímeros (ver Figura 4B) forma tetrámeros de agua (ver figura 5B).

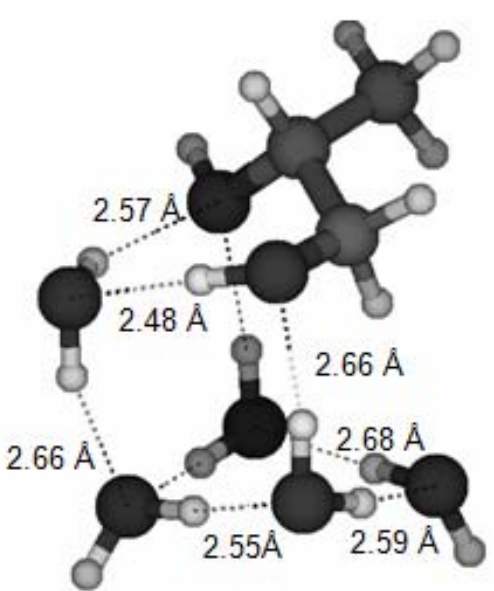

$4 \mathrm{~A}$

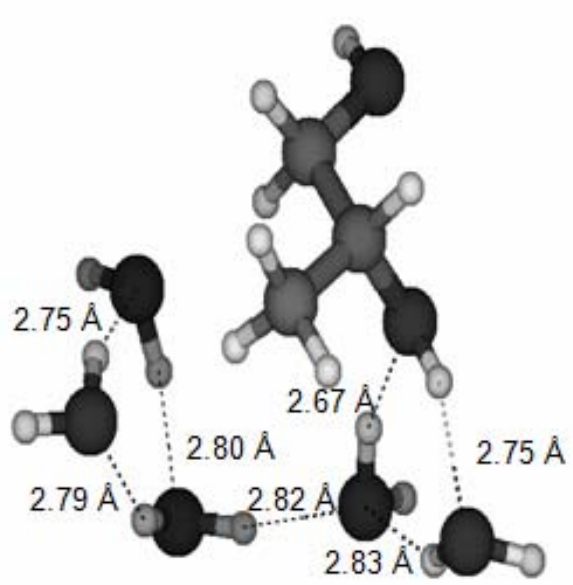

$4 \mathrm{~B}$

Fig. 4: Clústeres de mínima y máxima energía para el sistema 1,2-propanodiol con 5 moléculas de agua. Distancias $\mathrm{HO} \cdots \mathrm{H}$ en angstrom $(\AA)$ y ángulos de enlace $\mathrm{O}-\mathrm{H}-\mathrm{O}$ en grados $\left(^{\circ}\right)$

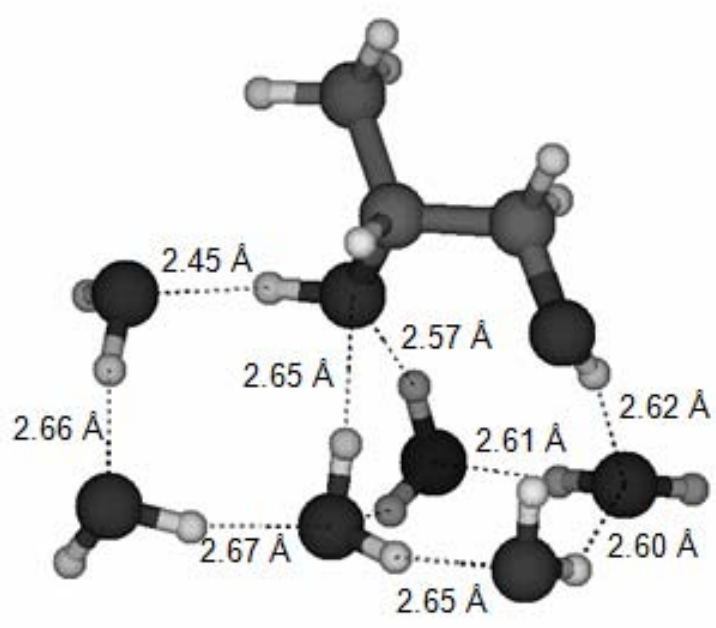

$5 A$

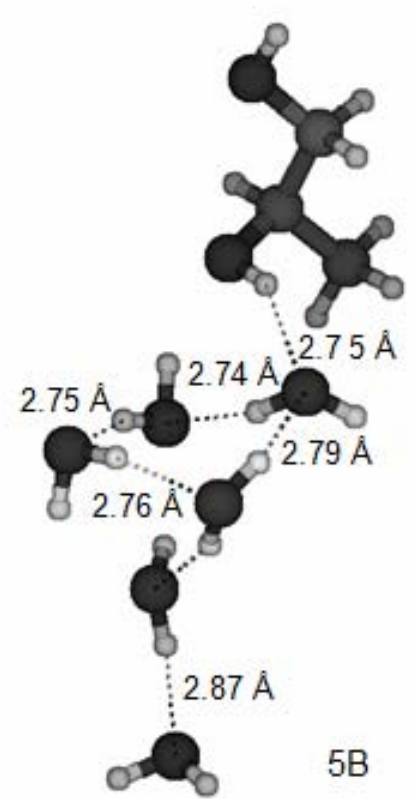

$5 \mathrm{~B}$

Fig. 5: Clústeres de mínima y máxima energía para el sistema 1,2-propanodiol con 6 moléculas de agua. Distancias $\mathrm{HO} \cdots \mathrm{H}$ en angstrom $(\AA)$ y ángulos de enlace $\mathrm{O}-\mathrm{H}-\mathrm{O}$ en grados $\left(^{\circ}\right)$ 


\section{CONCLUSIONES}

Las principales fuerzas de interacción para la formación de los clústeres con estructuras geométricas más estables fueron las que correspondieron a enlaces de hidrógeno primario del tipo $\mathrm{HO} \cdots \mathrm{H}$.

El sistema de 1,2-propanodiol y 3 moléculas de agua formaron el mayor número de clústeres debido a la menor interacción entre las moléculas de agua, favoreciendo las interacciones aguadiol sobre las interacciones agua-agua. Las preferencias geométricas y la alta estabilidad de las configuraciones para los mínimos globales son una consecuencia directa de la fuerza de los enlaces de hidrógeno del tipo primario y su arreglo espacial caracterizado por distancias de enlace de hidrógeno cortas y ángulos de enlace con tendencia hacia la colinealidad.

Los resultados presentados son un muy buen estimativo del estudio de las mezclas agua/dioles, aportando al entendimiento del comportamiento de estas mezclas al ser utilizadas como cosolventes en la industria farmacéutica.

La metodología escogida para este estudio resulta ser una aproximación razonable para inferir sobre las interacciones del 1,2-propanodiol y moléculas de agua, debido al uso de herramientas estocásticas para explorar la superficie de energía potencial donde se maximiza la ocurrencia de las configuraciones cercanas a los mínimos.

\section{AGRADECIMIENTOS}

Al Centro de Investigación Universidad de Córdoba (CIUC) por la financiación. Al Ph.D Albeiro Restrepo por el soporte del programa ASCEC. Al Químico Ramón Martínez por su arduo trabajo y aporte.

\section{REFERENCIAS}

Buggins,T.R., P.A. Dickinson y G. Taylor., The effects of pharmaceutical excipients on drug and disposition, Advanced Drug Delivery Reviews 59, 1482-1503 (2007).

Cuevas, G., Introducción a la química computacional, $1^{\mathrm{a}}$ edición, Fondo de Cultura Económica, México (2003).

David, J., D. Guerra y A. Restrepo., Structural Characterization of the (Methanol)4 Potential Energy Surface, J. Phys. Chem. A, 113, 10167-10173 (2009).

Dixit, S., A.K. Soper., J.L. Finne y J. Crain., Water structure and solute association in dilute aqueous methanol, Europhys. Lett., 59, 377-383 (2002).

Frisch, M., G.W., Trucks, y otros 80 autores; Gaussian03, Revision E.01, Gaussian, Inc, Wallingford CT, Pittsburgh, USA (2004).

Grabowski, S.J., W.A. Sokalski., y J. Leszczynski., How Short Can the H--H Intermolecular Contact $B e$ ? New Findings that Reveal the Covalent Nature of Extremely Strong Interactions, ChemInform 36 (2005).

Jimenez, J. y F. Martínez., Study of some volumetric properties of 1,2-propanodiol + water mixtures at several temperatures, Rev. Col. Cienc. Quím. Farm, 34, 46-57 (2005).

Koch, W., A chemist's guide to density functional theory, $2^{\mathrm{a}}$ edición. Wiley- $\mathrm{VCH}$, Weinheim, New York (2001).

Kong, J., y otros treintra y tres autores., Q-Chem 2.0: a high-performanceab initio electronic structure program package, J. Comput. Chem., 21, 1532-1548 (2000). 
Mejía, S.M., J.F. Espinal., A. Restrepo y F. Mondragón., Molecular Interaction of (Ethanol)2-Water Heterotrimers, J. Phys. Chem. A., 111, 8250-8256 (2007).

Mejía, S.M., J.F. Orrego., J.F. Espinal y F. Mondragón., Heteropentámeros (etanol)4-agua: estudio estructural y termodinámico, Quím. Nova, 33 (2010).

Oliveira, B. y M. Vasconcellos., Hydrogen bonds in alcohols water complexes: A theoretical study about new intramolecular interactions via CHELPG and AIM calculations, Journal of Molecular Structure: THEOCHEM 774, 83-88 (2006).

Perez, D., C. Guevara., C. Cárdenas y J. Pinzón., Solubilidad y volúmenes de desplazamiento del acetaminofen en mezclas binarias formadas por agua, etanol y propilenglicol a $25^{\circ} \mathrm{C}$, Rev. Col. Cienc. Quím. Farm. 32, 116-136 (2003).

Pérez, J.F., C.Z. Hadad y A. Restrepo., Structural studies of the water tetramer. Int. J. Quant. Chem., 108, 1653-1659 (2008).

Reichardt, C., Solvents and solvent effects in organic chemistry, $3^{\mathrm{a}}$ edición, Wiley-VCH, Weinheim (2003).

Restrepo, A., Influence of $\pi$ bonds on the structure of organic molecules, (Ph.D) (2005).

Romero, C.M., M.S. Páez., J.C. Arteaga., M.A. Romero y F. Negrete., Effect of temperature on the volumetric properties of dilute aqueous solutions of 1,2-hexanediol, 1,5-hexanediol, 1,6hexanediol, and 2,5-hexanediol, J. Chem. Thermodyn., 39, 1101-1109 (2007).

Ruckenstein, E., I.L. Shulgin y J.L. Tilson., Treatment of Dilute Clusters of Methanol and Water by ab Initio Quantum Mechanical Calculations, J. Phys. Chem. A., 109, 807-815 (2005).

Swarbrick, J., Encyclopedia of pharmaceutical technology, $2^{\mathrm{a}}$ edición, Marcel Dekker, New York (2002). 